\title{
Microsecond Pulsed Laser Material Ablation by Contacting Optical Fiber
}

\author{
Maxim N. SINYAVSKY ${ }^{* 1}$, Vitaly I. KONOV ${ }^{* 1}$, Taras V. KONONENKO ${ }^{* 1}$, Vladimir P. PASHININ ${ }^{* 1}$ \\ ${ }^{*}$ Natural Sciences Center, General Physics Institute, Vavilov str. 38, Moscow 119991, Russia \\ E-mail:kononen@nsc.gpi.ru
}

\begin{abstract}
The conditions that allow productive material ablation via contacting fiber, delivering microsecond pulses of Nd:YAG laser without damage of the fiber tip, are found. Stomatologic materials such as ceramics, composites and metal alloys were used as targets in atmospheric air. The choice of materials is caused by high potential of contacting fiber ablation technique in dentistry. Silica fiber with core diameter of $300 \mu \mathrm{m}$ was positioned at distances $\Delta=0-1 \mathrm{~mm}$ from the target surface. Pumpprobe laser shadowhraphy allowed to study the material plasma expansion dynamics and peculiarities (size, speed, direction of motion) of the target microparticles ejection. Two types of fiber tip damage were studied: catastrophic (mechanical destruction preventing further ablation) and acceptable (enhanced light absorption and scattering by the target material deposited on the fiber accompanied by the tip surface low rate etching by laser beam with formation of a minor negative lens). It is shown that for laser fluencies $\mathrm{E} \approx 20-40 \mathrm{~J} / \mathrm{cm}^{2}$, when microsecond pulsed materials ablation rates are quite high $(\sim 1-10 \mu \mathrm{m} /$ pulse $)$, for the investigated targets no catastrophic damage of contacting fiber tip occurs for all gaps and laser pulse number $\mathrm{N} \leq 10^{3}-10^{4}$, excluding metal case with $\Delta \leq 10-20 \mu \mathrm{m}$. DOI:10.2961/jlmn.2010.03.0008
\end{abstract}

Keywords: laser, ablation, fiber, damage, plasma, shadowhraphy

\section{Introduction.}

Many of material microprocessing operations require fiber delivery of pulsed laser radiation to the workplace. Moreover, some of them (e.g. laser ablation techniques in dentistry, endoscopic operations or surface cleaning) can be essentially improved if a fiber tip appears in the vicinity or even in direct contact with an object to be processed. $\mathrm{Nd}$ :YAG laser pulses at the fundamental wavelength (1.064 $\mathrm{nm}$ ) can be transmitted through conventional, large-core silica fibers. As laser intensity within a fiber is increased, however, transmission will eventually be interrupted by laser-induced damage. One of the key laser parameters affecting the damage threshold fluence is pulsewidth. For widely used Q-switched Nd:YAG lasers ( $\tau=10 \mathrm{~ns}$ ), typical damage threshold is close to $10^{2} \mathrm{~J} / \mathrm{cm}^{2}$, but it increases to the value of $10^{3} \mathrm{~J} / \mathrm{cm}^{2}$ for microsecond pulses and reaches more than $10^{5} \mathrm{~J} / \mathrm{cm}^{2}$ for millisecond pulses [1]. Other critical issues which determine the laser-induced damage threshold include the laser mode and launch conditions, index profile, fiber connectors and, especially, end finish. It is known [2] that a good mechanical or $\mathrm{CO}_{2}$ laser polish applied to the fiber faces can increase the damage threshold by more than a factor of two when compared to a fiber with a poor end finish. Hence, one can expect that probable appearance of even small defects on the fiber face contacting with the ablated material should facilitate dramatic reduction of the damage threshold.

The aim of this work was to find microsecond-pulsed $\mathrm{Nd}$ :YAG laser parameters allowing fast material ablation by contacting silica fiber without catastrophic damage of the fiber tip by the laser radiation and ablation products. It was shown earlier [3] that microsecond laser ablation is a good compromise between high ablation rate and precision of pulsed laser material processing. "Catastrophic damage" of the fiber tip in the investigated case means that the transmitted radiation fluence drops down below material ablation threshold. In other words, modification of the fiber tip, which does not cause a noticeable reduction of the ablation rate within a certain pulse number, is considered as an acceptable result.

Contacting fiber ablation technique was studied mostly, for the particular case of laser ablation of dental materials. As we have demonstrated earlier [4], laser radiation with a wavelength of $1064 \mathrm{~nm}$ and microsecond pulse duration is a promising tool for dental applications. It allows effective ablative cleaning of the tooth surface from various dental materials in the absence of damage to dental tissues (dentine, enamel). Naturally, with contacting fiber ablation this technique can be essentially advanced.

\section{Experimental procedure}

Three kinds of stomatologic materials were used in the experiments as targets: self-curing composites with fillerparticle size of about $1 \mu \mathrm{m}$ (Herculite XRV, Kerr, Germany), zirconia ceramics (Zircad, Ivoclar Vivadent), and Fe-based alloys. Laser pulses with $\tau=1.3 \mu \mathrm{s}$, energy $200 \mathrm{~mJ}$ and repetition rate $\mathrm{f}=1-100 \mathrm{~Hz}$ were generated by a Nd:YAG laser with a fiber-optic delay line inside its cavity [3]. Laser radiation was delivered to the samples by another quartz fiber with core diameter of $300 \mu \mathrm{m}$ coupled to the laser output.

Two irradiation schemes were utilized in the experiments. In the remote ablation mode, the output tip of the delivering fiber was positioned at a large distance from the sample. The image of the tip surface was projected by a lens on the sample surface into a spot with $100 \mu \mathrm{m}$ diameter. This irradiation mode was applied to measure the material ablation rates and to investigate parameters of the laser produced plasma. In the contact ablation mode, the air gap $\Delta$ between the delivering fiber tip and the sample surface varied in the range of $0 \div 1 \mathrm{~mm}$. 
Time-resolved images of the various phases of material ablation were obtained with a pump-probe diagnostic set-up. The probe beam from a Q-switched Nd:YAG laser ( $\tau=10 \mathrm{~ns})$ after frequency doubling $(\lambda=532 \mathrm{~nm})$ and collimation was sent tangentially to the sample surface to illuminate the air region where laser interaction and material removal occurred. The probe laser was emitted with delay of $\tau_{d}=100 \mathrm{~ns} \div 10 \mu \mathrm{s}$ in respect to the moment when intensity of the microsecond laser pulse reached $10 \%$ of the maximum value. The ablation zone illuminated by the probe beam was imaged by an objective into the entrance of a CCD camera coupled to a frame grabber. Shadowgraphic images allowed reliable detection of shock wave fronts, microscopic objects (droplets, particles), ejected from the target surface, and simplified control of the gap value $\Delta$. To enhance visibility of the plasma plume, which was found very transparent for the probe beam, Schlieren technique was applied. A knife-edge positioned at the back focal plane of the objective filtered out probe light passed through the ablation zone without refractive index assisted perturbations.

The delivering fiber was cleaved without any following treatment of the fiber face. Preservation of the fiber functionality under multishot material processing in the contact mode was estimated from control of the ablation rate data. Depth of the produced craters, as well as the fiber face morphology before and after material processing was evaluated with optical and white-light interference microscopy.

\section{Results}

The damage threshold for the multimode fibers delivering $1.3 \mu \mathrm{s}$ laser pulses without target ablation was found close to the expected level [1]. The highest value was obtained under cleaving of the fiber faces $\left(E_{d}=550 \div 600 \mathrm{~J} / \mathrm{cm}^{2}\right)$, while mechanical polishing of the faces caused reduction of the parameter - down to $E_{d}=450 \div 500 \mathrm{~J} / \mathrm{cm}^{2}$. As it follows from the ablation rate curves on Fig.1, ablation thresholds for all materials studied are in the range $E_{a}=10 \div 30 \mathrm{~J} / \mathrm{cm}^{2}$, i.e. the condition $E_{a}<<E_{d}$ is fulfilled. Note, the ablation rates could be as high as $60 \mu \mathrm{m} /$ pulse for the composite material and $5 \div 10 \mu \mathrm{m} / \mathrm{shot}$ for the metal alloy and ceramics irradiated at the fluence $E \leq 100 \mathrm{~J} / \mathrm{cm}^{2}$, which is still essentially lower than the fiber damage threshold.

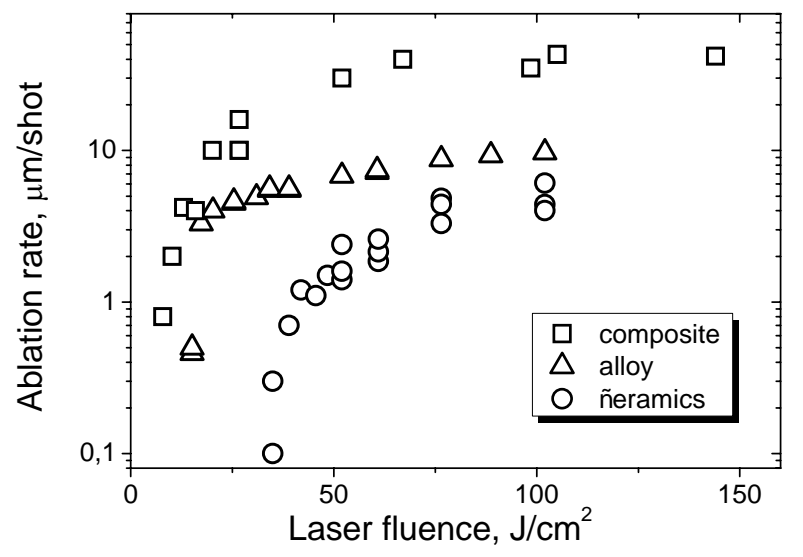

Fig.1 Ablation rates for different materials.

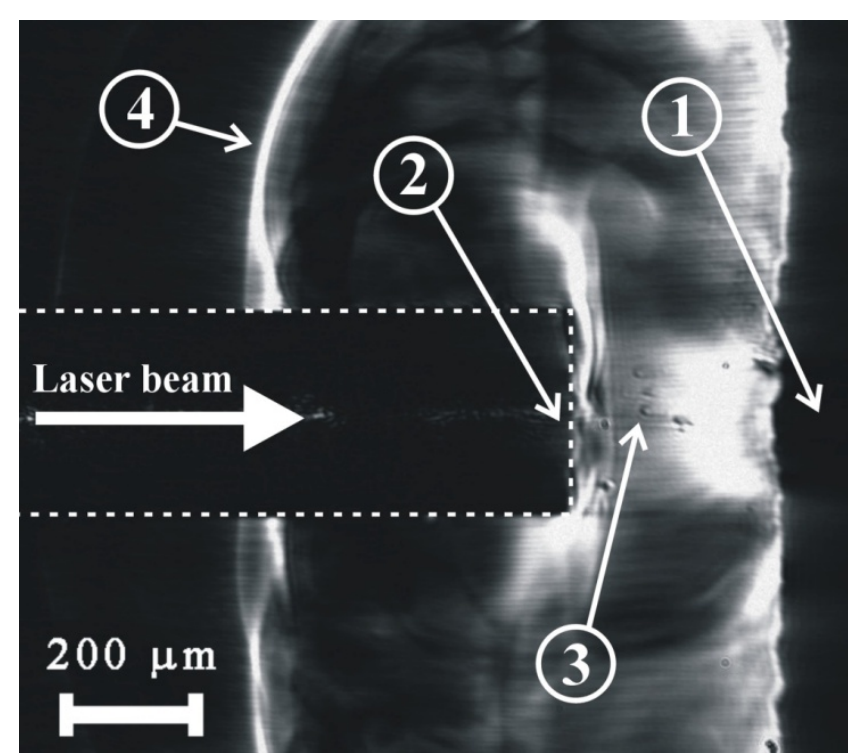

Fig. 2. Shlieren photo of metal alloy ablation in contact mode $\left(E=30 \mathrm{~J} / \mathrm{cm}^{2}, \tau_{d}=1.9 \mu \mathrm{s}\right): 1$ - target, 2 - fiber tip, 3 - ejected microparticles, 4 - plasma plume front.

Effect of the alloy target ablation on the contacting fiber is illustrated by a Shlieren photo in Fig.2 (time delay $\tau_{\mathrm{d}}=1.9 \mu \mathrm{s}$ ). The laser radiation passes through the fiber from the left to the right and vaporizes the target material, producing plasma in the expanding vapor plume. The plasma plume can interact with the fiber tip in different ways including plasma etching, which modifies the fiber face profile, and deposition of vaporized target material, which leads to reduction of the fiber face transmission. The microparticles, that where, ejected from the laser heated surface and reached the fiber tip, also could cause its damage or become attached to the fiber face. The shock wave formed at the front of the expanding plasma must be taken into account as well.

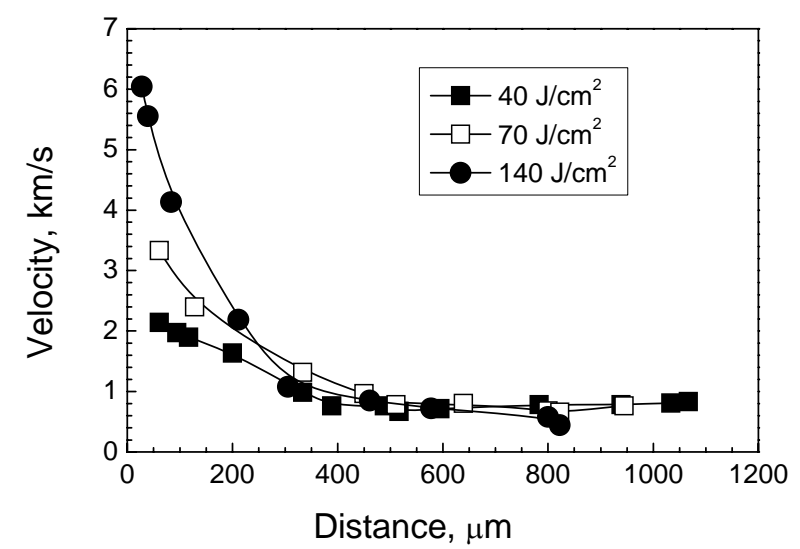

Fig.3. Velocity of plasma front as a function of distance from ceramics target.

Analyzing displacement of the plasma front on the Slieren photos depending on the time delay, we have evaluated the front velocity as a function of distance $r$ from the irradiated surface (for instance, $V(r)$ for the ceramic target in Fig.3). The plasma front propagates with supersonic speed, which reduces from $2 \div 6 \mathrm{~km} / \mathrm{s}$ near the target surface down to $600 \div 800 \mathrm{~m} / \mathrm{s}$ at the distance of more than 
$0.5 \mathrm{~mm}$. This leads to formation of a shock wave easily identified on the Shlieren photos due to sharp change in the refraction index. The vapor pressure behind the shock wave can be evaluated from the experimental data on $V(r)$ and the limiting formula for strong shock wave [5]:

$$
P_{s w}=\frac{2}{\gamma+1} A^{2}
$$

where $\gamma \approx 1.2$ - Poisson coefficient, $\rho_{0}$ - density of undisturbed air. The calculated vapor pressure $P_{s w}(r)$ presented in Fig.4, reaches $40 \div 300$ bar near the target surface for laser fluence ranging from 40 up to $140 \mathrm{~J} / \mathrm{cm}^{2}$. Close results were obtained also for the alloy and composite targets.

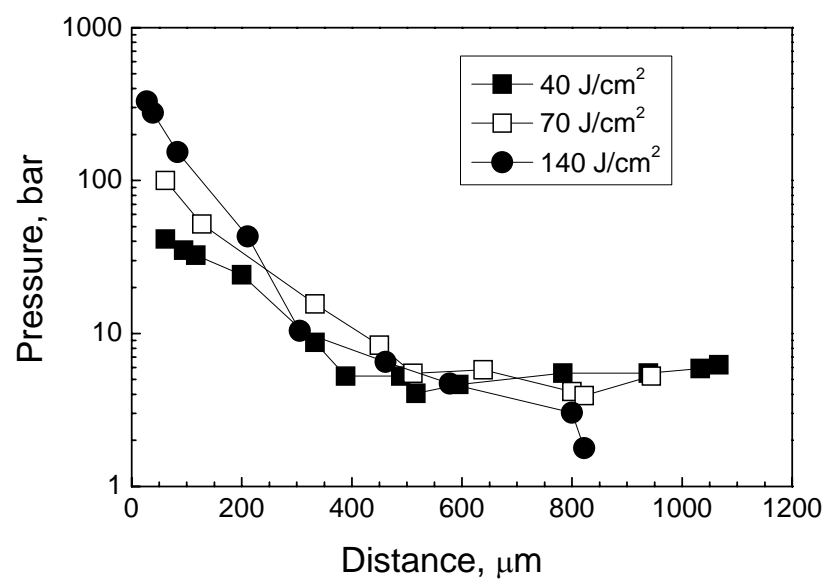

Fig.4. Pressure behind the plasma front as a function of distance from ceramics target.

Comparison of our data with the results of similar shadowgraphic investigation performed for shorter (6 ns) and longer ( $20 \mu \mathrm{s}$ and $200 \mu \mathrm{s}$ ) laser pulses [6] makes possible to specify changes in the plasma parameters under variation of pulsewidth. In particular, the plasma produced by microsecond pulses $(\tau \geq 1.3 \mu \mathrm{s})$ is found highly transparent for $1064 \mathrm{~nm}$ wavelength in contrast to the opaque nanosecond plasma. Rise of the pulse duration in the mentioned range leads to progressive reduction of the typical plasma pressure from 400 bar $(6 \mathrm{~ns})$ to 40 bar $(1.3 \mu \mathrm{s})$ and further to $\sim 1$ bar for $20 \mu$ s pulses under comparable values of incident fluence $\left(20 \div 40 \mathrm{~J} / \mathrm{cm}^{2}\right)$.

Ablation of all examined targets was accompanied by pronounced ejection of microparticles depending on the target material nature. In the case of both metal and ceramic targets, essential part of the material was removed as liquid droplets moving with the speed of about $50 \div 100 \mathrm{~m} / \mathrm{s}$ at small angles (about $20^{\circ}$ ) to the target surface (Fig.5). To our opinion, such a behavior is determined by motion of liquid material along the sample surface due to radial gradient of vapor recoil pressure estimated at the level of $\sim 10^{4} \mathrm{bar} / \mathrm{cm}$.

For composite targets, the size of ejected particles could be much larger (up to $100 \mu \mathrm{m}$ ) as it follows from Fig.6. The fragments moved normally to the target surface with much higher speed $\leq 700 \mathrm{~m} / \mathrm{s}$ that is close to the plasma expansion velocity. This fact correlates with extremely high ablation rates for the composite (up to $60 \mu \mathrm{m}$ per shot) that can be seen on Fig.1. We attribute this effect to a peculiar mechanism of ablation of composite materials
- volume explosion. High transparency of base composite material leads to localization of the laser pulse energy deep in the sample rather at the surface. The heating area can be localized at grain interfaces where radiation absorption is higher. Such a mechanism is also supported by microscopic investigation of laser produced craters. While for metal (ceramic) materials crater and spot diameters are approximately equal, on composite samples crater diameter, a result of bulk explosion, could be several times greater than the diameter of irradiation spot.

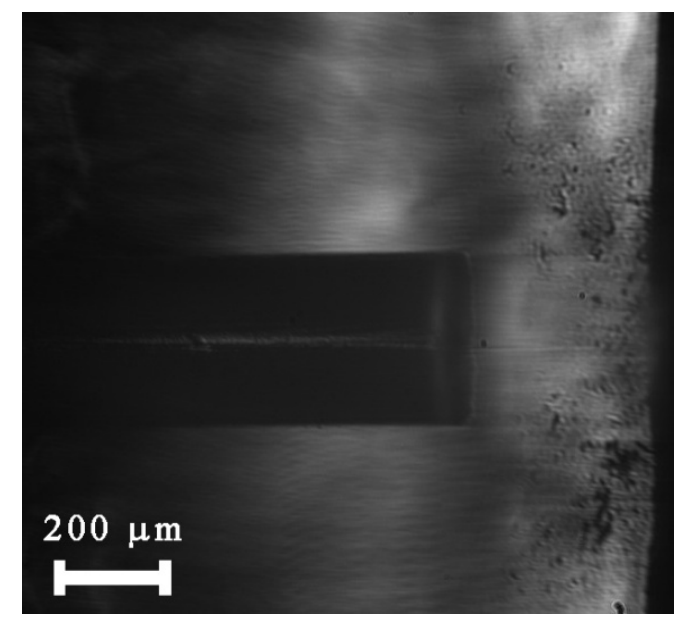

Fig.5. Ejection of microparticles under ceramic ablation $\left(E=35 \mathrm{~J} / \mathrm{cm}^{2}, \tau_{d}=3.4 \mu \mathrm{s}\right)$

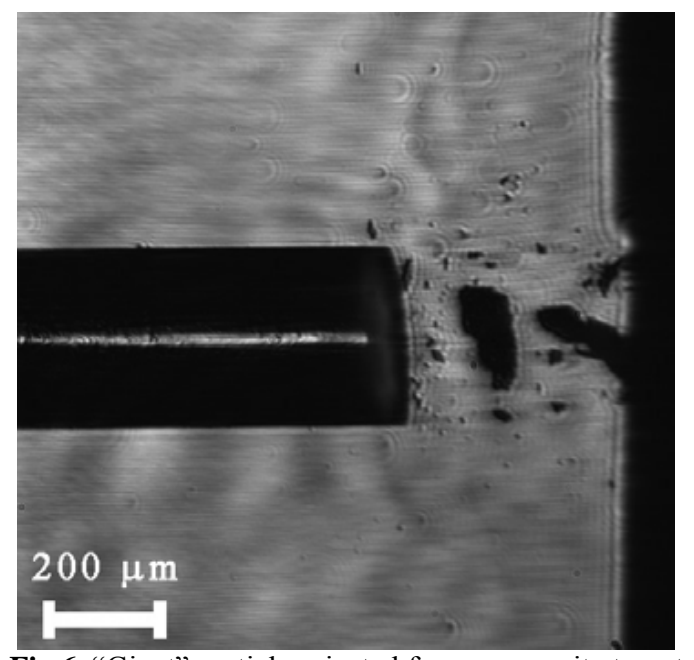

Fig.6. "Giant" particles ejected from composite target $\left(E=40 \mathrm{~J} / \mathrm{cm}^{2}, \tau_{d}=5 \mu \mathrm{s}\right)$

The feature of the metal target ablation under close contact between the fiber and the target surface $(\Delta \leq 10$ $20 \mu \mathrm{m}$ ) was catastrophic damage of the fiber tip already after few or even a single pulse $\left(E \geq 20 \mathrm{~J} / \mathrm{cm}^{2}\right)$. The observed destruction (see Fig.7) seems to be explained by impact of melted metal jets. Elimination of this effect at slight increase of the air gap can be the result of either fragmentation of the massive jets onto smaller droplets or ejection of the melted material at sliding angle without impact with the fiber tip. Let us emphasize that multishot $\left(N \leq 10^{3} \div 10^{4}\right)$ contact fiber ablation at other conditions within the mentioned limits (metal for $\Delta>10-20 \mu \mathrm{m}$, other targets, $\left.\Delta=0 \div 1 \mathrm{~mm}, E \leq 40-100 \mathrm{~J} / \mathrm{cm}^{2}\right)$ caused no functional destruction of the fiber, which would affect the ablation 
rate. However, we have revealed certain non-crucial modifications of the contacting fiber face.

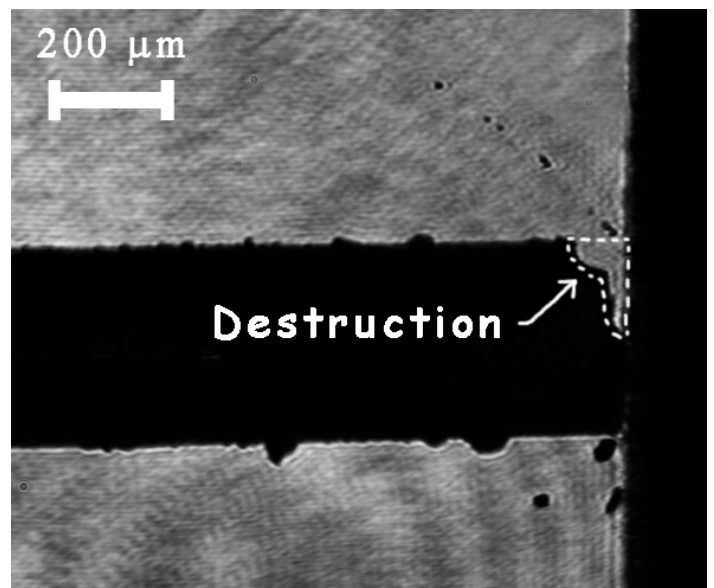

Fig.7. Destruction of the fiber tip under close contact $(\Delta \approx 0)$ with metal alloy target $\left(E=35 \mathrm{~J} / \mathrm{cm}^{2}\right)$.

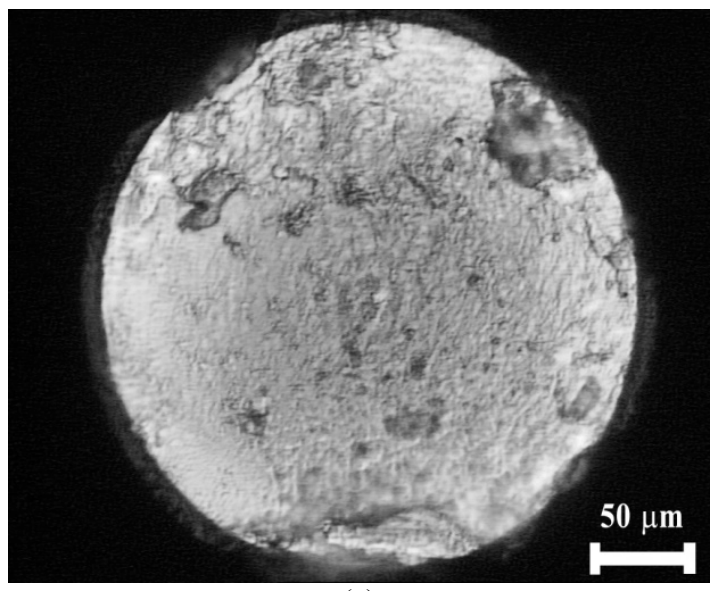

(a)

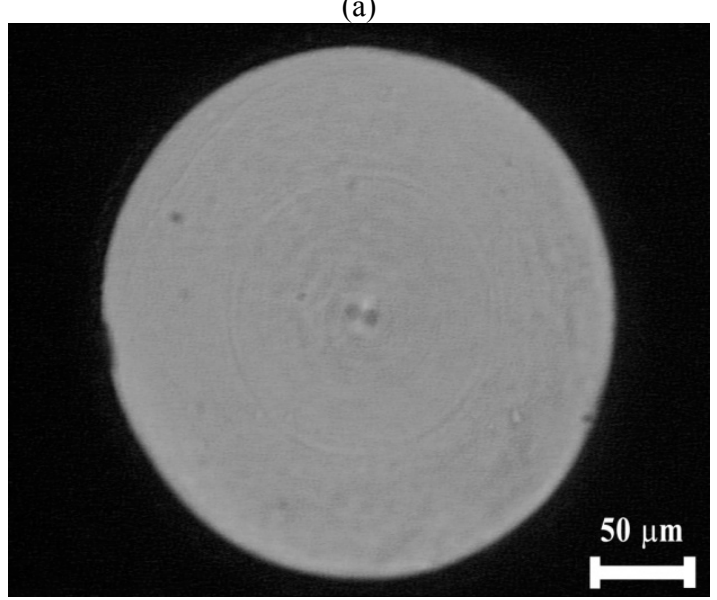

(b)

Fig.8. Microscopic photos of the fiber tip after multishot ablation in contact mode of different targets: (a) composite, (b) alloy $\left(\Delta=300 \mu \mathrm{m}, 300\right.$ shots, $\left.E=35 \mathrm{~J} / \mathrm{cm}^{2}\right)$

After ablation of the composite target, the fiber face is covered by numerous ablated fragments of different size (Fig. 8a). On the contrary, no deposited target material appears on the fiber face after contact ablation of the metal alloy and ceramic target (see Fig. 8b). In the last case, however, pronounced modification of the fiber face profile was found. Appearance of a shallow crater at the fiber face
(Fig.9) indicates preferable etching of the fiber core. Experiments on contact ablation with a small air gap $(\Delta=20 \mu \mathrm{m})$ controlled by the shadowgraphic imaging prove that the etching rate of the fiber cladding is at least two orders of magnitude lower than typical etching rate of the fiber core. This indicates a decisive role of the laser radiation, which passes mainly though the fiber core.

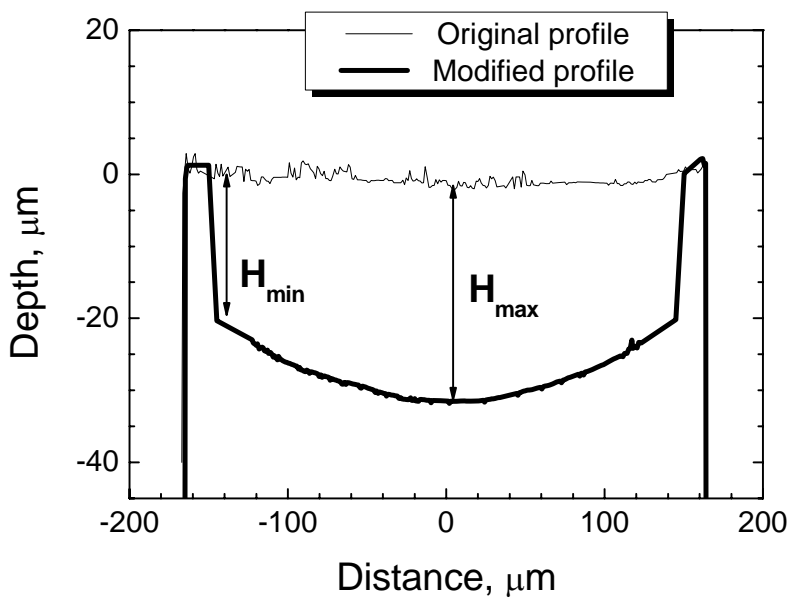

Fig.9. Transformation of the fiber tip profile after multishot ablation of metal target in contact mode $(\Delta=500 \mu \mathrm{m}, 300$ shots,

$$
E=35 \mathrm{~J} / \mathrm{cm}^{2} \text { ) }
$$

To explain the observed modification of the fiber tip, one should take into account an additional phenomena revealed under time resolved shadow visualization of the contact ablation. Figure 10 shows formation of a small plasma plume at the fiber face before the ceramics (alloy) target ablation begins. The observed plume results from ablation of the material deposited on the fiber face during few preceding laser pulses. Typically, $1 \div 5$ laser shots were required to produce an opaque layer, which could be heated and vaporized by the laser radiation. This secondary plasma does not influence the target ablation, but causes gradual removal of silica at the fiber face. Deposition of the ablated material (vapor and particles) observed under the composite target processing, however, does not lead to detectable etching of the fiber face due to, presumably, low absorption of the material.

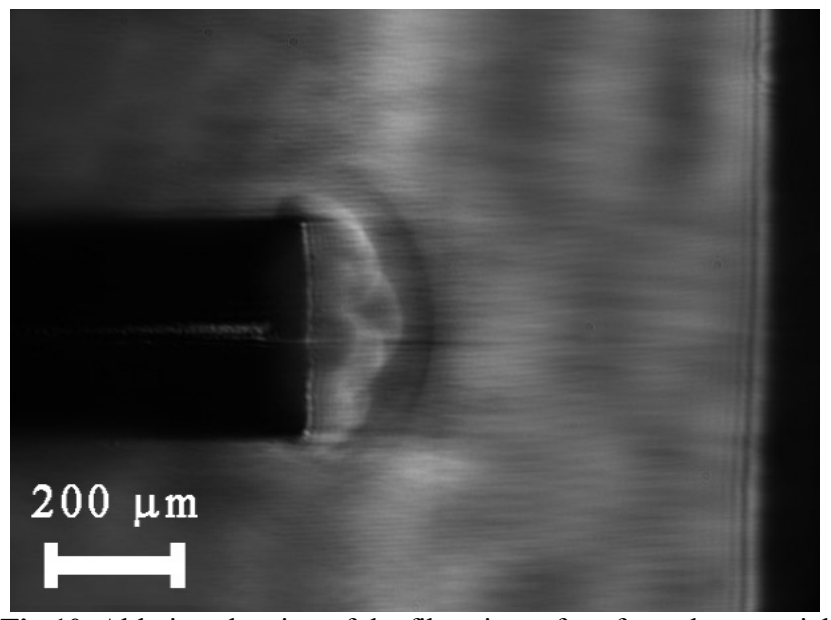

Fig.10. Ablative cleaning of the fiber tip surface from the material deposited by previous pulses (ceramics, $E=35 \mathrm{~J} / \mathrm{cm}^{2}, \tau_{d}=0.7 \mu \mathrm{s}$ ). 
The fact that the deposited layer is ablated from the fiber tip surface by the laser beam and not by expanding plasma of the target material is supported by the special experiment we carried out. Two identical fibers were stacked together. No radiation was passing through the extra (passive) fiber though the target material was deposited on its surface with practically equal rate. And it was found that the passive fiber surface profile remained flat, only slightly roughened, while the core of the laser beam transmitting fiber had a concave profile as a result of multiple pulsed irradiation.

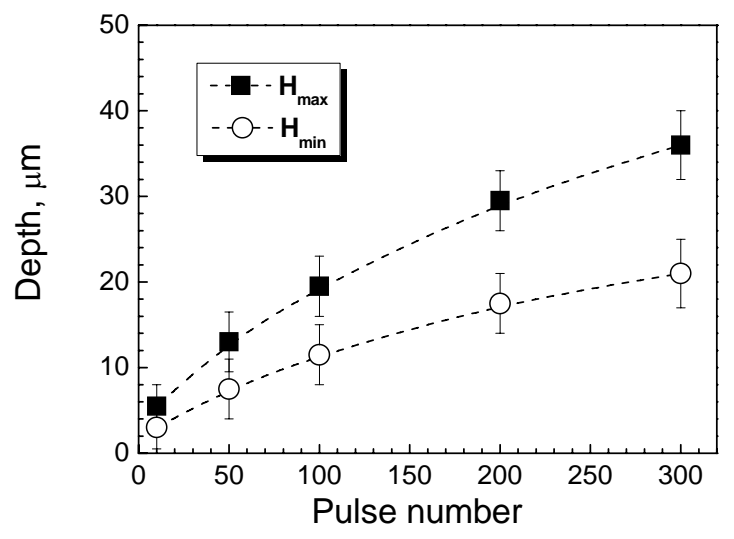

Fig.11. Parameters of modified fiber tip profile as a function of laser pulse number (alloy, $\Delta=300 \mu \mathrm{m}, E=35 \mathrm{~J} / \mathrm{cm}^{2}$ ).

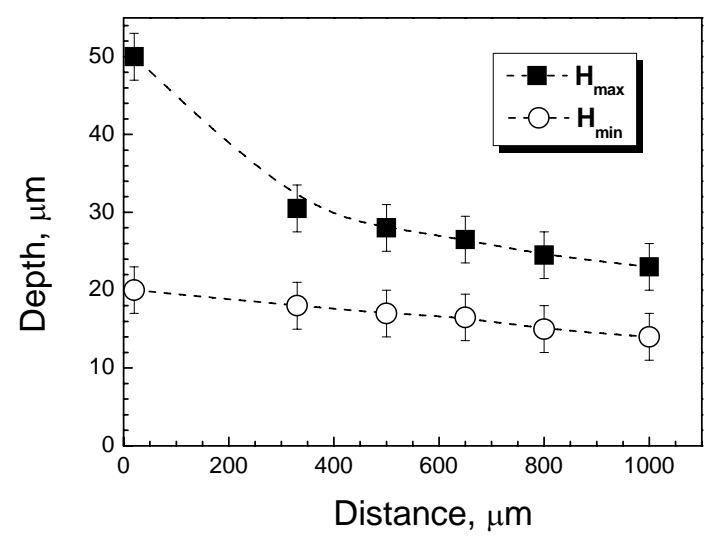

Fig.12. Parameters of modified fiber tip profile as function of distance from the target surface (alloy, 200 shots, $E=35 \mathrm{~J} / \mathrm{cm}^{2}$ ).

Figure 11 demonstrates progressive transformation of the basic fiber face profile characterized by maximum and minimum crater depths $\left(H_{\max }\right.$ and $H_{\min }$ in Fig.9). The depth of the fiber core concave profile $H_{\max }-H_{\min }$ increased with the laser pulse number but never exceeded $\approx 30-40 \mu \mathrm{m}$ for $N \leq 10^{4}$ and $E=30-40 \mathrm{~J} / \mathrm{cm}^{2}$. Such $H_{\max }-H_{\min }$ values are much smaller than the fiber diameter and could not decrease beam intensity dramatically for the investigated gaps $\Delta$ and, correspondingly, could not prevent further target contact ablation. Naturally, as it follows from Fig.12 the effect of fiber tip etching was most pronounced for small gaps $\Delta<300 \mu \mathrm{m}$ and maximum $H_{\max }-H_{\min } \approx 30 \mu \mathrm{m}$ values were achieved when $\Delta \rightarrow 0$ (in the case the concave lens the fiber tip radius of curvature is about $300 \div 400 \mu \mathrm{m}$ ), while for
$\Delta \approx 1 \mathrm{~mm}$ the value of $H_{\max }-H_{\min }$ was about $10 \mu \mathrm{m}$. More intensive fiber tip etching at small gaps can be explained by the contact with hot plasma volume.

An interesting effect was found for drilling of the composite material by contacting quartz fiber. The dependence of the crater depth on laser pulse number $N$ for different $E$ is presented on Fig.13. One can easily see that for small $E \leq 30 \mathrm{~J} / \mathrm{cm}^{2}$ saturation of ablation process takes place for pulse number $N>10-20$, when the crater depth was equal to the irradiation spot diameter. The ultimate crater depth decreases for smaller $E$ and is only about $10-20 \mu \mathrm{m}$ for $E=15 \mathrm{~J} / \mathrm{cm}^{2}$ which is close to composite ablation threshold (see Fig.1). We consider that this effect is the result of vapor plasma confinement in the crater by the contacting fiber and target surface shielding by such plasma. For $E \geq 50 \mathrm{~J} / \mathrm{cm}^{2}$ (Fig.13) a normal linear dependence of the crater depth with $N$ was observed which can be explained by essentially enhanced crater diameters induced by the composite laser explosion mechanism [4].

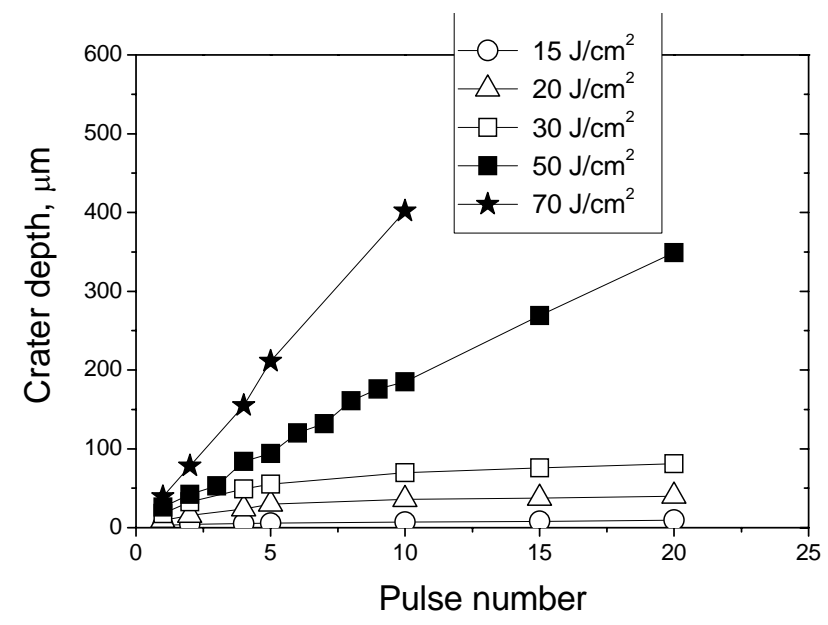

Fig.13. Crater depth produced by contacting fiber in composite sample for various irradiation pulse number

The peculiarity of contact fiber ablation of materials is demonstrated on Fig.14. The through channel was produced in ceramic sample with $850 \mu \mathrm{m}$ thickness by the quartz fiber with removed protective polymer cladding. For pulsed laser fluence $E \approx 50 \mathrm{~J} / \mathrm{cm}^{2}$ only 50 laser pulses were required to produce the channel, while special tests have shown that for such a material and moderate E levels the catastrophic damage did not occur in contacting ablation mode for $N \leq 10.000$.

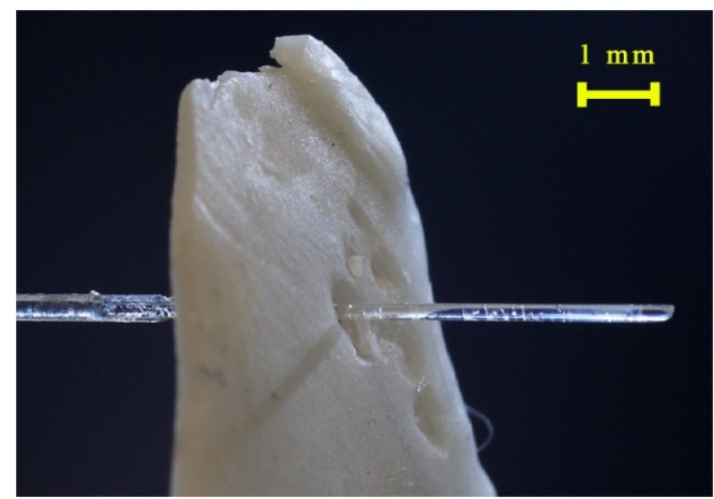

Fig. 14. Through drilling of composite with contact fiber ablation. 


\section{Conclusions.}

It has been experimentally shown that various materials (ceramics, composites, metals) can be effectively ablated by microsecond pulses of Nd:YAG laser in fiber contacting mode. Only metal targets require gaps $(\Delta \geq 10-20 \mu \mathrm{m})$ between the fiber tip and their surface to avoid the fiber catastrophic damage. For other materials in close contacting mode $(\Delta=0)$ and $\Delta \geq 10-20 \mu \mathrm{m}$ for metals high rate $(1-$ $10 \mu \mathrm{m} / \mathrm{shot}$ or even greater) ablation can be realized for $E \leq 50-100 \mathrm{~J} / \mathrm{cm}^{2}$ and irradiation pulse number $N \leq 10^{3}-10^{4}$.

The obtained data opens the possibility to perform productive and precise removal of different stomatologic materials from the tooth tissue by a flexible contacting fiber technique as well as improve some other important surface cleaning operations.

\section{Acknowledgments}

The work was supported by Russian Federal Agency for Science and Innovation (02.740.11.0417, SS$7556.210 .2)$.

\section{References}

[1] R.M. Wood: Third International Workshop on Laser Beam and Optics Characterization, Quebec City, Proc.SPIE, 2870, (1996) 457.

[2] R.E. Setchell: Laser-Induced Damage in Optical Materials, Boulder, Proc.SPIE, 2966, (1997) 608.

[3] S.V. Garnov, V.I. Konov, T.V. Kononenko, V.P. Pashinin and M.N. Sinyavsky: Laser Physics, 14, (2004) 910.

[4] M.L. Siniaeva, M.N. Siniavsky, V.P. Pashinin, Ad.A. Mamedov, V.I. Konov and V.V. Kononenko: Laser Physics, 19, (2009) 1056.

[5] Ya.B.Zel'dovich, and Yu.P.Raizer: "Physics of shock waves and high-temperature hydrodynamic phenomena", ed. by W.D.Hayes, R.F.Probstein (Academic Press, New York and London, 1966) v.1, p.94

[6] S. Siano, F. Margheri, R. Pini, P. Mazzinghi and R. Salimbeni: Appl. Opt., 36, (1997) 7073. 